\title{
Understanding Solvent/Bixin Interactions by Raman Spectroscopy
}

\author{
Lenize F. Maia, ${ }^{a}$ Isabela M. R. Rimulo, ${ }^{a}$ Vanessa E. de Oliveira,${ }^{b}$ Júlio A. F. Arvellos, ${ }^{c}$ \\ Luiz A. S. Costa, ${ }^{\circledR c}$ Howell G. M. Edwards ${ }^{d}$ and Luiz F. C. de Oliveira ${ }^{\circledR *, a}$ \\ ${ }^{a}$ Núcleo de Espectroscopia e Estrutura Molecular (NEEM), Departamento de Química, \\ Instituto de Ciências Exatas, Universidade Federal de Juiz de Fora, \\ 36036-900 Juiz de Fora-MG, Brazil \\ ${ }^{b}$ Departamento de Ciências da Natureza, Instituto de Humanidades e Saúde, \\ Universidade Federal Fluminense, Campus Rio das Ostras, 28895-532 Rio das Ostras-RJ, Brazil

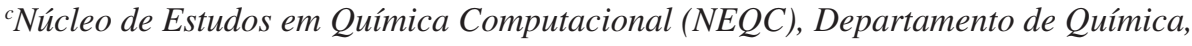 \\ Instituto de Ciências Exatas, Universidade Federal de Juiz de Fora, \\ 36036-900 Juiz de Fora-MG, Brazil \\ ${ }^{d}$ School of Chemistry and Biosciences, Faculty of Life Sciences, University of Bradford, \\ BD7 1DP Bradford, West Yorkshire, United Kingdom
}

\begin{abstract}
In this work, Raman spectroscopy and density functional theory (DFT) calculations have been used in order to understand the nature of the interaction between the carotenoid bixin and organic solvents with dielectric constant varying from ca. 2 (toluene) to ca. 47 (dimethyl sulfoxide). Raman shifts registered for the main carotenoid marker band, the $\mathrm{C}=\mathrm{C}$ stretching mode $\left(1525-1533 \mathrm{~cm}^{-1}\right)$, were used as a probe to monitor spectral changes due to chemical interaction between bixin in the solid state with the analogous solvated species. Raman spectra of bixin in solution showed significant bathochromic shifts in the wavenumber position of $v(\mathrm{C}=\mathrm{C})$ when compared with the solid state. Among the solutions, subtle differences were observed and the polarizability rather than dielectric constant of the solvents seems to be a more appropriate parameter to explain the differences in Raman shifts which could be correlated with the solvation degree of bixin. Correlation between DFT analysis and molecular dynamics simulations obtained for cis-bixin in DMSO and $\mathrm{CHCl}_{3}$ demonstrated that the interaction of the solute with the respective solvents occurs in specific portions of the molecule, however, this result was not confirmed by the experimental data since bixin is solvated by larger numbers of solvent molecules.
\end{abstract}

Keywords: carotenoids, bixin, solvents, Raman spectroscopy

\section{Introduction}

Carotenoids comprise carotenes (hydrocarbons), xanthophylls (with oxygen-containing functional groups) and apocarotenoids which are oxidative cleavage products of the $\mathrm{C}_{40}$ backbone skeleton. Carotenoids play an important role in distinctive biological processes, such as light-harvesting antenna and photo-protecting agents, both involved in photosynthesis. ${ }^{1-3}$ In such complex media, it is expected that carotenoids would interact through exposure to different chemicals; these molecular environmental interactions usually lead to changes in the spatial conformation of the polyenic chains, which consequently affect their electronic

*e-mail: luiz.oliveira@ufjf.edu.br properties. ${ }^{4,5}$ It is well known that solvents can exert a significant influence on the spectroscopic properties of carotenoids, especially those which affect their electronic delocalization, light absorption or scattering. ${ }^{3,6,7}$

The identification of carotenoids by the observation of specific vibrational modes of the polyenic chain utilized as molecular key markers has been extensively reported in the literature ${ }^{8-10}$ since the 1970 's. Their structures, involving the extended pi conjugation between the $\mathrm{C}=\mathrm{C}$ and $\mathrm{C}-\mathrm{C}$ bonds which results in the coupling of many molecular modes, give rise to elegant and simple vibrational spectra; three Raman bands are normally highlighted, namely, the stretching of the $\mathrm{C}=\mathrm{C}\left(v_{1}\right), \mathrm{C}-\mathrm{C}\left(v_{2}\right)$ bonds and the $\mathrm{C}-\mathrm{CH}_{3}$ in-plane group rocking vibrations $\rho\left(\mathrm{C}-\mathrm{CH}_{3}\right)$, occurring respectively at ca. 1520,1160 and $1000 \mathrm{~cm}^{-1} .7,11-13$ 
An important correlation has been observed between the $v_{1}$ wavenumber and the respective number of $\mathrm{C}=\mathrm{C}$ conjugated bonds in the polyene structure; $;^{14,15}$ numerous papers in the literature have used this empirical approach to characterize the presence of different carotenoids in diverse biological systems, such as plants, ${ }^{16}$ microorganisms, ${ }^{17}$ the shells of molluscs, ${ }^{18}$ pearls ${ }^{19}$ and octocorals. ${ }^{20-23}$ Recently, de Oliveira et al. ${ }^{13}$ used Raman spectroscopy in order to demonstrate that this approach must be exercised with caution, since these molecules are sensitive to their chemical environment, leading to misinterpretation and a misunderstanding of the occurrence of a particular carotenoid in an organic or mineral matrix. The environmental effect has also been discussed in an earlier study of several inclusion compounds of selected carotenoids and beta-cyclodextrin in which the influence of the surrounding molecules upon the $v_{1}$ mode of the carotenoid produced wavenumber shifts greater than $10 \mathrm{~cm}^{-1}$ in the carotenoid inclusion compound compared with that found in the free carotenoid species. ${ }^{24}$ The interaction of solvents with linear carotenes (e.g., lycopene) and carotenes containing terminal ionone rings (e.g., $\beta$-carotene, lutein) has been reported in the literature. ${ }^{5,25,26}$ The combination of a Raman spectroscopic and an absorption study of carotenoids has been used to discriminate between the polarizability effects and observed changes in the effective conjugation length of both types of carotenoids. ${ }^{25}$ However, corresponding studies performed with apocarotenoids has not been undertaken hitherto and in this work we have investigated the electronic and vibrational properties of cis-bixin (Figure 1), the 6-methyl hydrogen (9Z)-6,6'-diapocarotene$6,6^{\prime}$-dioate, which is the major constituent present in urucum or annatto seeds (Bixa orellana), with the chemical formula $\mathrm{C}_{25} \mathrm{H}_{30} \mathrm{O}_{4}$ accounting for ca. $80 \%$ of the carotenoid-derived pigments. ${ }^{27}$ The unique C-25 carbon skeleton here lacks terminating rings (e.g., $\beta$-ionone) and has been biosynthesized from the oxidative cleavage of a C-40 carotenoid skeleton. ${ }^{27}$ cis-Bixin is more polar than other carotenoids, exhibiting a carboxylic acid group at one end and a methyl ester group at the opposite end of the molecule, which confers some liposolubility to the pigment. ${ }^{28,29}$ It is therefore soluble in most polar organic solvents, conferring an orange coloration to the solution, but is largely insoluble in vegetable oil ${ }^{29}$ and aprotic apolar solvents. ${ }^{30}$

Seeds and powder form are commercially available as annatto and their main orange-red colored component is referred to as bixin (cis-bixin or Z-bixin). This compound has been extensively used as a food colorant ${ }^{27,28}$ with a wide range of pharmacological activities ${ }^{31,32}$ and due to its physicochemical properties it is used as a sensitizer for dye sensitized solar cells. ${ }^{33,34}$

It is known from literature that Raman spectroscopy is the molecular spectroscopic technique of choice for investigating carotenoids; Raman and electronic absorption experiments have been commonly used to explain the behavior of carotenoid molecules in different environments such as solvents, ${ }^{26,35}$ proteins, ${ }^{36}$ tissues $^{36}$ and mimetic systems. ${ }^{24,37}$ However, Raman spectral investigations of the apocarotenoid bixin has not been studied so far and the main purpose of this work is to evaluate the structural and spectroscopic changes incurred upon the interaction of bixin with different solvents. This study will also demonstrate the degree of susceptibility to which such an apocarotenoid is influenced by its chemical environment.

Previous work ${ }^{38}$ has shown that the resonance Raman spectrum of pure cis-bixin has major Raman bands at 1525, 1155 and $1010 \mathrm{~cm}^{-1}$. However, the behavior observed for this compound in solution is very intriguing, since for different solvents the Raman bands appear shifted, mainly the $v_{1}$ mode, which seems to be more susceptible to the molecular interaction with its chemical environment. To support the experimental measurements, theoretical DFT (density functional theory) calculations and molecular dynamics simulations were carried out in order to explain the molecular behavior of the apocarotenoid and its interaction with solvents having different dielectric constants and polarizabilities. Such results will reveal a more accurate perception of the interaction between carotenoids/polyenes and polar or non-polar solvents, which can contribute towards a better understanding of apocarotenoids in specific environments.

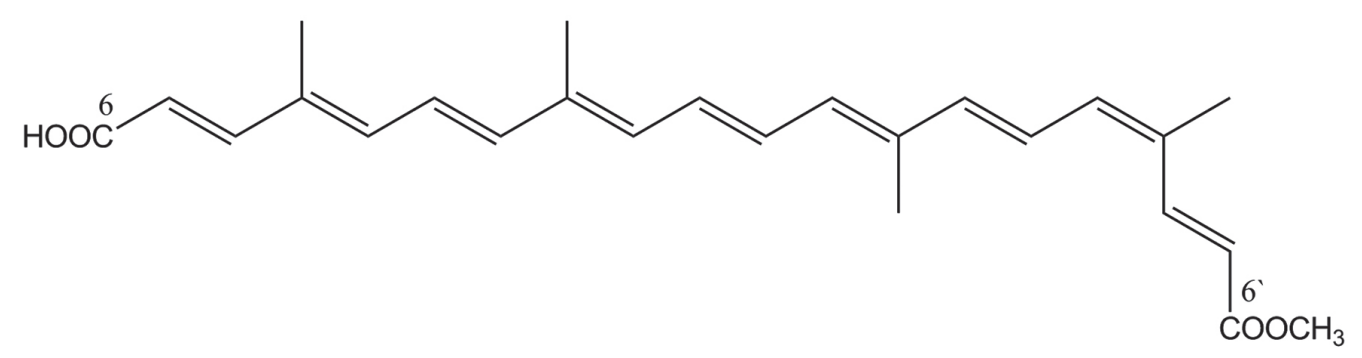

Figure 1. Chemical structure of cis-bixin (1), the 6-methyl hydrogen (9Z)-6,6'-diapocarotene-6,6'-dioate, the major constituent present in urucum or annatto seeds (Bixa orellana). 


\section{Experimental}

Bixin was extracted from seeds of Bixa orellana according to the following procedure: $25.0 \mathrm{~g}$ of seeds were washed twice with $50 \mathrm{~mL}$ of hexane for $15 \mathrm{~min}$ with stirring; after filtration, the seeds were washed with methanol in a similar procedure. The methanolic extract was evaporated under reduced pressure, yielding an orangered solid, comprising more than $90 \%$ of cis-bixin, according to de Oliveira et al. ${ }^{38}$

UV-Visible spectra were obtained using a Shimadzu UVPC-1601 spectrophotometer in seven different solvents: chloroform $\left(\mathrm{CHCl}_{3}\right)$, dichloromethane $\left(\mathrm{CH}_{2} \mathrm{Cl}_{2}\right)$, toluene ( $\mathrm{PhMe}$ ), nitromethane $\left(\mathrm{CH}_{3} \mathrm{NO}_{2}\right)$, dimethyl sulfoxide (DMSO, $\left(\mathrm{CH}_{3}\right)_{2} \mathrm{SO}$ ), acetonitrile $\left(\mathrm{C}_{2} \mathrm{H}_{3} \mathrm{~N}\right)$ and pyridine $\left(\mathrm{C}_{6} \mathrm{H}_{5} \mathrm{~N}\right)$ (all spectroscopic grade, purchased from SigmaAldrich, St. Louis, USA), from 200 to $1100 \mathrm{~nm}$ with a $2 \mathrm{~nm}$ spectral resolution, using $10 \mathrm{~mm}$ quartz cuvettes, using $2 \mathrm{~nm}$ as scan rate.

The solid cis-bixin obtained from the methanolic extract, as well the solutions from each the solvents mentioned above, were analyzed by Raman spectroscopy using a Bruker RFS 100 FT-Raman instrument equipped with a germanium detector refrigerated by liquid nitrogen. Laser excitation at $1064 \mathrm{~nm}$ was provided from an Nd:YAG (neodymium-doped yttrium aluminum garnet) laser with a power at the sample of $200 \mathrm{~mW}$, in the range between $3500-100 \mathrm{~cm}^{-1}$, with a spectral resolution of $2 \mathrm{~cm}^{-1}$ and an average of 512 scans accumulated. These parameters were selected in order to achieve better signal-to-noise ratios for which the physical and chemical integrity of the samples could be preserved; the spectral analyses were performed in duplicate.

\section{Theoretical calculations}

In order to provide feasible information related to the structures of bixin in different solvents, theoretical calculations have been performed. Optimizations and harmonic frequency calculations were carried out under the formalism of DFT. Grimme's ${ }^{39,40}$ B97 pure functional method including dispersion has been chosen for these calculations using Pople and co-workers' ${ }^{\prime 4}$ basis set $6-311 \mathrm{G}(\mathrm{d}, \mathrm{p})$. The vibrational frequencies were calculated in order to simulate the observed Raman spectra for each compound. All thermal contributions were set at $1 \mathrm{~atm}$ and $298.15 \mathrm{~K}$. The continuum method IEF-PCM (integral equation formalism-polarizable continuum model) has been used for the optimizations. ${ }^{42,43}$ All the quantum mechanical calculations reported here were carried out with the Gaussian 09 program $^{44}$ and the NEQC (Núcleo de Estudos em Química Computacional, Universidade Federal de Juiz de Fora, Juiz de Fora, Brazil) servers.

The explicit investigation of the interactions between the solvent and the cis-bixin molecule was conducted using molecular dynamics (MD) simulations on Amber12.45 Solvent molecules have initially been optimized on Gaussian $09^{44}$ as unitary molecules (in vacuum) using a B3LYP/6-31G(d) level of theory. The simulations were carried out with Amber12 software ${ }^{45-47}$ using a generalized AMBER force field field (GAFF) to parametrize cisbixin and the AM1-BCC (Austin model 1-bond charge correction) charges were obtained with the antechamber package of Amber. Also, a correction for atomic types has been applied (as explained by referral to atom type GAFF in Sprenger et al. . ${ }^{46}$ The standard test solvent has been made by using water TIP3PBOX. Chloroform solvent is already available in Amber, but dimethyl sulfoxide (DMSO) parameters were obtained from Fox and Collman. ${ }^{48}$ After parametrizing cis-bixin, it was solvated with about 3500 solvent molecules, the system, cis-bixin and its chosen solvent (either DMSO or $\mathrm{CHCl}_{3}$ ), was then minimized without any restraint and heated in one step from 9 to $300 \mathrm{~K}$ under the NVT ensemble, as shown in Figures S8 and S9 (Supplementary Information (SI) section, for DMSO only). After heating, the equilibration and following production were carried out under the NPT ensemble ( 1 bar and $300 \mathrm{~K}$ ). In all steps of the simulations, the SHAKE algorithm was employed, removing all hydrogen bond stretching. The production was carried out for a 1 ns simulation after system equilibration, which provides a sufficient insight into the first solvation shell, which was obtained from cpptraj analysis, as described in the Results and Discussion section.

\section{Results and Discussion}

Since the goal of this work is an attempt to provide an understanding of the bixin behavior in selected solvents, it is important to display their electronic spectra. Figure 2 shows the electronic spectra of bixin in several different solvents such as: chloroform, dichloromethane, toluene, nitromethane, dimethyl sulfoxide, acetonitrile and pyridine, all of them containing the same concentration of carotenoid (approximately $10^{-5} \mathrm{~mol} \mathrm{~L}^{-1}$ ). Three peaks can be clearly seen in the electronic spectrum, representing the vibrational fine structure of the $\mathrm{S}_{0} \rightarrow \mathrm{S}_{2}$ transition (the $1{ }^{1} \mathrm{~A}_{\mathrm{g}} \rightarrow 1{ }^{1} \mathrm{~B}_{\mathrm{u}}$ transition); these peaks arise from electronic transitions from the lowest vibrational level of the electronic ground state to the lowest vibrational levels of the electronic excited state.

It is important to note that the shape of the electronic transitions of bixin changes when the solvent is changed. 


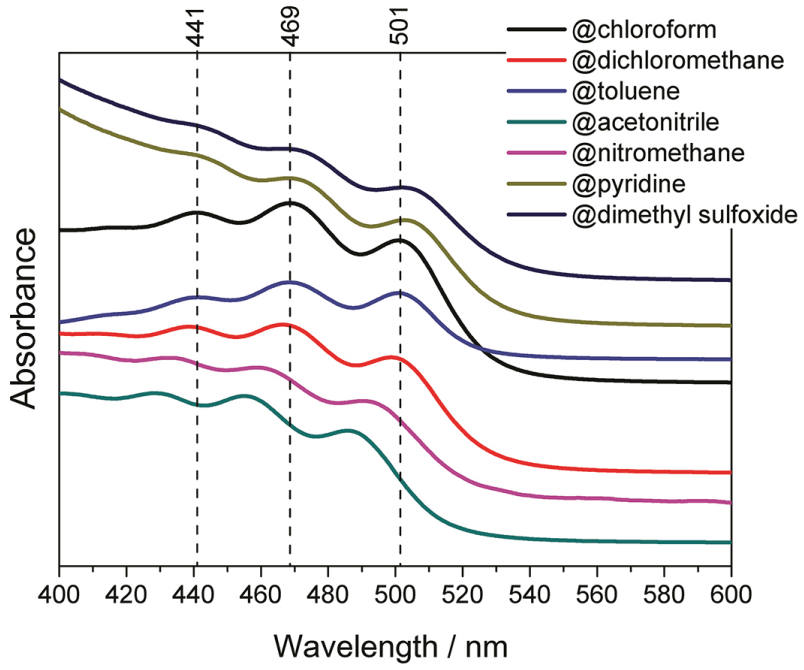

Figure 2. Electronic spectra of bixin in different solvents.

For example, for solvents with high values of the dielectric constant such as DMSO (value of 46.8), the electronic transitions can be seen at $502 \mathrm{~nm}$, one of the highest wavelength values observed here. However, the dielectric constant does not seem to be the fundamental property which determines the peak position since the solution in toluene, with a significantly lower dielectric constant (value of 2.38), has shown an electronic band observed at $501 \mathrm{~nm}$. Furthermore, the electronic spectrum obtained with other solvents investigated does not show any correlation with the polarity of the solvent. Britton et al..$^{49}$ showed that the UV spectral shift of carotenoids in solution depends on the polarizability rather than the polarity of the solvent; as the refractive index of the solvent increases a spectral shift to longer wavelength is observed. Recently, Rahmalia et al. ${ }^{30}$ showed that the $\mathrm{S}_{0} \rightarrow \mathrm{S}_{2}$ transition energy of bixin in solution was dependent mainly on the refractive index of the solvents and the bixin-solvent dispersion interaction. The electronic behavior of bixin in solvents with different polarities experiences a different chemical environment, however, it has been shown that a slight perturbation of the polyenic electronic distribution occurs when the solute is in contact with the solvent molecules. From our experimental data, it can be seen that the solution of bixin in acetonitrile has an electronic transition at $486 \mathrm{~nm}$, the lowest value for this band observed in this work; yet this solvent presents a dielectric constant value of 37.5 , the second-highest value of all the solvents investigated here. These data can be ascribed to a very unusual behavior where the interactions between the bixin and the solvent host occur in different portions of both the carotenoid and the solvent molecules. In cases where the solvent possesses at least one relatively polar portion of the molecular structure, such a moiety will be the one that would interact preferentially with the similar polar region of the molecular structure of the conjugated polyene, thereby attempting to minimize the competition between the intermolecular interaction forces, even if these are relatively very weak. Certainly, this type of interaction affects the solubility of the carotenoid in the respective solvents; in some cases, it is almost impossible to dissolve the solid in the required solvent and only a very small portion of the polyene interacts effectively.

Such behavior can be also seen in the Raman spectra depicted in Figure 3, where the comparison between the bixin spectra in the solid state with the analogous acetonitrile solution has been provided. The main Raman bands observed in the apocarotenoid spectrum do not differ much from carotenoids containing the $\beta$-ionone ring, such as carotene. ${ }^{50}$ Solid bixin exhibits a strong band at $1520 v_{1}(\mathrm{C}=\mathrm{C}), 1155 v_{2}(\mathrm{C}-\mathrm{C})$ and $1012 \mathrm{~cm}^{-1} \rho\left(\mathrm{C}-\mathrm{CH}_{3}\right)$. However, the bixin in our chosen seven selected solvents all show a red shift of the $v^{1}(\mathrm{C}=\mathrm{C})$ band. Macernis et al. ${ }^{26}$ observed that the strength of valence bonds in several carotenoids changes in the presence of solvents, thus modulating the $\pi$-electronic system in the $\pi$-conjugated chain. Results obtained here from bixin in acetonitrile have shown a band shift from $1520 \mathrm{~cm}^{-1}$ observed in the solid sample to $1533 \mathrm{~cm}^{-1}$ in solution (Figure 3). Theoretical Raman spectra of cis-bixin in acetonitrile and in the gas phase using the IEF-PCM method are shown in Figure 4; they are quite similar, with major differences occurring in Raman bands occurring in the wavenumber range of $1750-1650 \mathrm{~cm}^{-1}$ which have been attributed to the $v(\mathrm{C}=\mathrm{O})$ mode; other important parameters can be seen in Table S1, provided as Supplementary Information data. This observation is highly relevant considering that such a mode would be related to the chemical interaction of solvents with polar groups at both ends of the cis-bixin structure. The presence of bands at 1723 and $1705 \mathrm{~cm}^{-1}$ in the calculated spectrum in a solution of acetonitrile could be correlated to the experimental data in the same solvent and in the solid sample, showing a weak broad band at 1711 and $1718 \mathrm{~cm}^{-1}$, respectively (Figures 3 and 4). The difference of band position for the $\mathrm{C}=\mathrm{O}$ stretching mode between the solid and the solute is about $7 \mathrm{~cm}^{-1}$, while for the $\mathrm{C}=\mathrm{C}$ stretching mode is $13 \mathrm{~cm}^{-1}$. The conjugated system in the linear backbone of the bixin seems to be a better site of interaction with acetonitrile than the polar C-terminal groups. The presence of hydroxyl group in the acid moiety may favor the $\mathrm{H}$-aggregation of bixin in acetonitrile due to a blue shift in the optical absorption maximum as reported by Tay-Agbozo et al..$^{51}$ In analogy to other carotenoids, such as lycopene, ${ }^{52}$ astaxanthin ${ }^{53}$ and zeaxanthin ${ }^{54}$ the differences of $v(\mathrm{C}=\mathrm{C}), v(\mathrm{C}-\mathrm{C})$ Raman shifts may reflect aggregate conformations. 


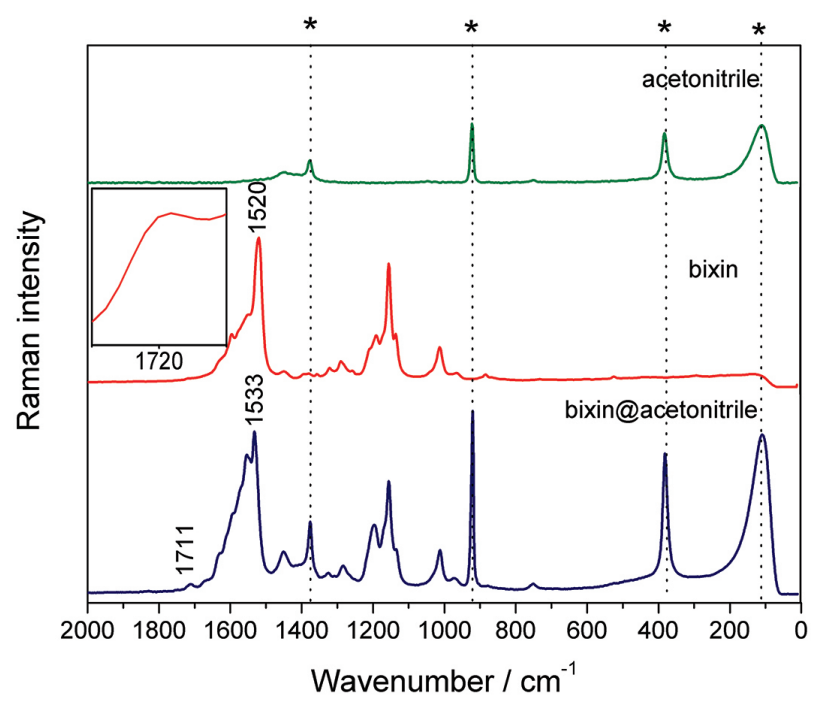

Figure 3. Comparison of Raman spectra of bixin in acetonitrile (blue line), the solid bixin (red line) and the pure solvent (green line). The stars indicate the solvent bands.

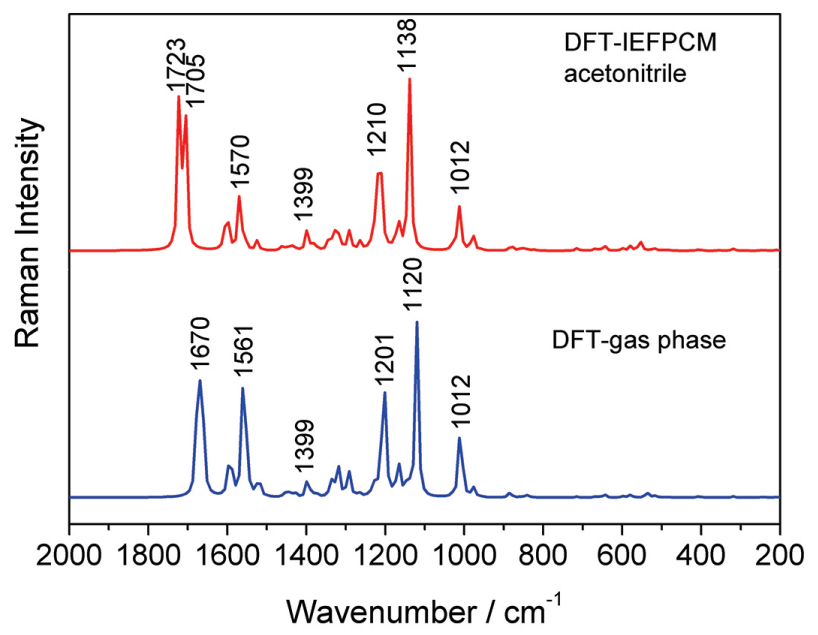

Figure 4. Comparison between calculated Raman spectra of cis-bixin in acetonitrile and gas phase.
Figures S1 to S7 given in the Supplementary Information section display the experimental Raman spectra of all the seven solutions studied here, as well as the spectra of the pure solvents and of the solid bixin for comparison purposes. It is well known from the literature ${ }^{24}$ that the $\mathrm{C}=\mathrm{C}$ stretching vibration $\left(v_{1}\right)$ is very sensitive to the chemical environment and that this is responsible for the deviation of its observed wavenumber position, whereas the other bands are not affected by the molecular surroundings. In this sense, the analysis of the bands listed in Table 1 shows clearly this behavior: the bands at 1155 and $1012 \mathrm{~cm}^{-1}$, describing the $v_{2}(\mathrm{C}-\mathrm{C})$ and $\rho\left(\mathrm{C}-\mathrm{CH}_{3}\right)$ modes, are not easily disturbed by the solvent interactions.

In a recent revision, discussing the electronic and vibrational properties of carotenoids from in vitro to in vivo, Llansola-Portoles et al. ${ }^{56}$ presented a very interesting relationship among the value of the $\mathrm{S}_{0} \rightarrow \mathrm{S}_{2}$ electronic transition and the value of the $\mathrm{C}=\mathrm{C}$ stretching bond (the $v_{1}$ mode) with the polarizability of several different solvents. ${ }^{56}$ There is a linear correlation between such parameters, and this could describe how the electronic and vibrational properties are related to the molecular configuration of carotenoids. Figure 5 shows a similar relationship for bixin with all the solvents we have used here, and the trends are clearly denoting the correlation between polarizability with electronic and vibrational data. Figure 5 a shows the plotting of the wavenumber of the electronic transition in each one of the used solvents against the calculated polarizability solvent defined as $R(\eta)=\left(\eta^{2}-2\right) /\left(\eta^{2}+1\right), \eta$ being the refractive index of the solvent ${ }^{56}$ Figure $5 \mathrm{~b}$ shows the plotting of the wavenumber of the $v_{1}$ vibrational mode versus the solvent polarizability $\mathrm{R}(\eta)$. In both graphics, it can be seen a correlation between these properties, but it is more evident when we take into account the electronic transition, rather than the vibrational description. This can be explained as the pi electrons, which are responsible

Table 1. Main vibrational modes from bixin in different solvents evaluated according to the dielectric constants and polarizability values

\begin{tabular}{|c|c|c|c|c|c|c|}
\hline \multirow{2}{*}{ Solvent/sample } & \multirow{2}{*}{ Dielectric constant $(\varepsilon)$} & \multirow{2}{*}{ Polarizability $^{\mathrm{a}}(\alpha)$} & \multirow{2}{*}{$\mathrm{UV}$-Vis / nm } & \multicolumn{3}{|c|}{ Vibrational mode $/ \mathrm{cm}^{-1}$} \\
\hline & & & & $v_{1}$ & $v_{2}$ & $\rho_{3}$ \\
\hline Solid bixin & - & - & - & $1520 \mathrm{~s}$ & $1155 \mathrm{~s}$ & $1010 \mathrm{w}$ \\
\hline Acetonitrile & 35.688 & 4.44 & 486 & $1533 \mathrm{~m}$ & $1156 \mathrm{~m}$ & $1012 \mathrm{w}$ \\
\hline Nitromethane & 35.562 & 4.97 & 490 & $1531 w$ & 1156w & - \\
\hline Dichloromethane & 8.93 & 6.52 & 499 & $1528 \mathrm{~s}$ & $1157 \mathrm{~m}$ & $1010 \mathrm{w}$ \\
\hline Dimethyl sulfoxide & 46.826 & 8.03 & 502 & $1529 m$ & 1156w & - \\
\hline Chloroform & 4.7113 & 8.53 & 501 & $1527 \mathrm{~s}$ & $1157 \mathrm{~m}$ & $1010 \mathrm{w}$ \\
\hline Pyridine & 12.978 & 9.58 & 502 & $1529 m$ & $1156 w$ & - \\
\hline Toluene & 2.3741 & 12.4 & 501 & $1529 \mathrm{~s}$ & $1157 \mathrm{~m}$ & $1010 \mathrm{w}$ \\
\hline
\end{tabular}

abtained from Bosque and Sales. ${ }^{55}$ Raman spectral intensities: w: weak; m: medium; s: strong. 
for the electronic transitions, are more disturbed by the surrounding media, the solvent, than the vibrational movement, which does not depend only on the pi electrons. In other words, the electronic transition depends on the electronic excited state, whereas the Raman band depends more exclusively on the electronic ground state, in terms of wavenumber position. On the other hand, Figures $5 \mathrm{c}$ and $5 \mathrm{~d}$ show the dependence between the electronic and vibrational wavenumbers for bixin within the solvent polarizabilities $(\alpha)$, one of the possible solvent characteristics that can be used to see the changes in the structure for carotenoids. The results are almost the same, when comparing Figures 5a and $5 \mathrm{~b}$ with $5 \mathrm{c}$ and $5 \mathrm{~d}$; this points out to the fact that for bixin, a C9 structure, the interaction with the solvent medium is based on the solvent polarizability. Finally, looking at graphics it can be seen that the aromatic solvents, toluene and pyridine, do not fit very well within the solvent properties and the spectroscopic data (vibrational and electronic); such results are also in agreement with the ones reported by Andersson et al. ${ }^{57}$ For example, the linear
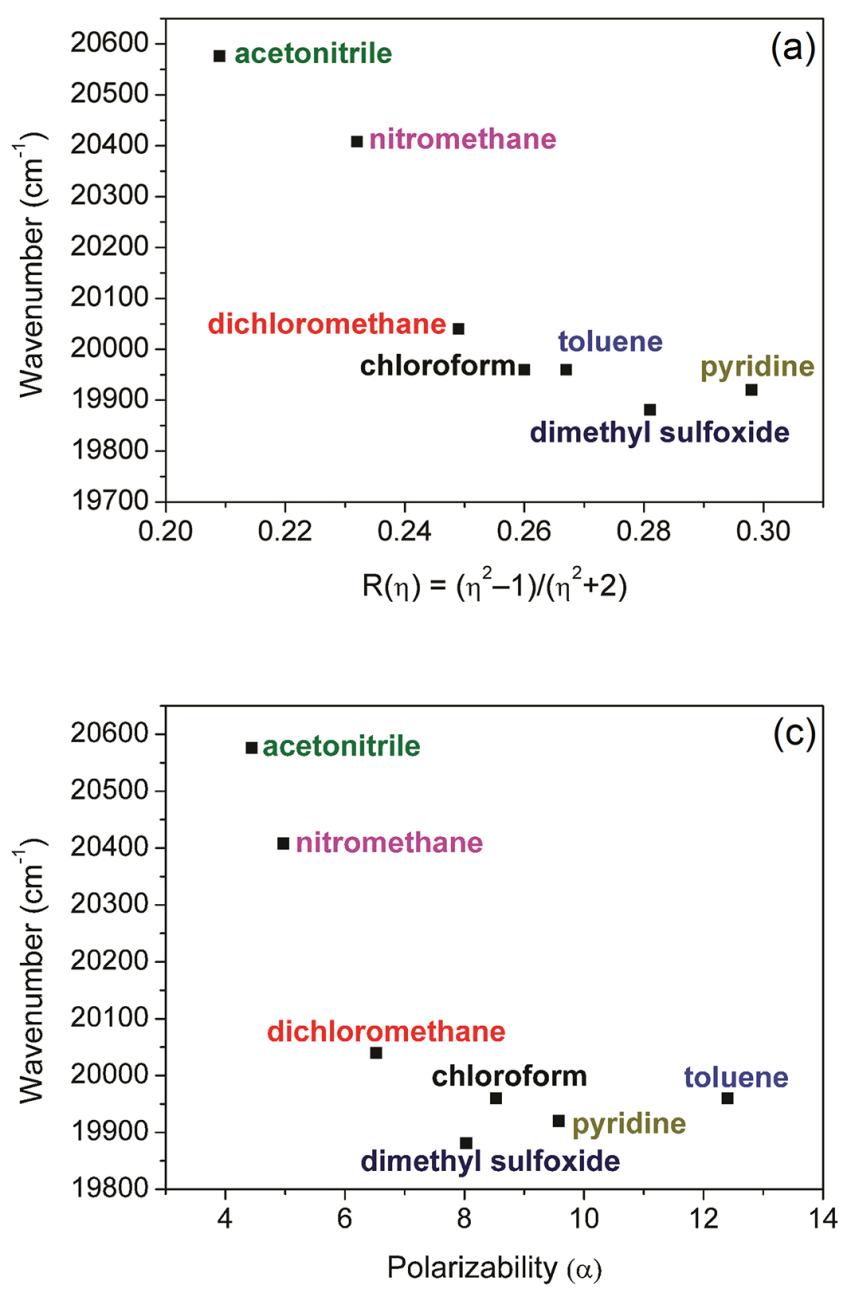

fit for Figure 5a, without one aromatic solvent (Figure S8, SI section), shows a linear adjustment coefficient of 0.91, which can be considered a very good result. The set of results shown here describes the influence of polarizability in the behavior of bixin dissolved in different media.

A detailed analysis of the Raman spectra of cis-bixin has been carried out in an earlier investigation, where resonance Raman spectra were used to characterize the electronic transition of the conjugated system..$^{38}$ Based on the use of the transform method equation, de Oliveira et al..$^{38}$ showed a variation in pattern of the bond length in the main trans-carbon backbone of cis-bixin in chloroform, which is the solvent that best solubilizes the solid. It was observed that the bond length changes were more pronounced in the middle of the carbon chain and these results indicated that just a small portion of the polyenic backbone presents an effective delocalized electronic charge; such data oppose the widespread idea that the electronic charge is equally delocalized over the whole structure of all the conjugated chain. It may be hypothesized that the small variation
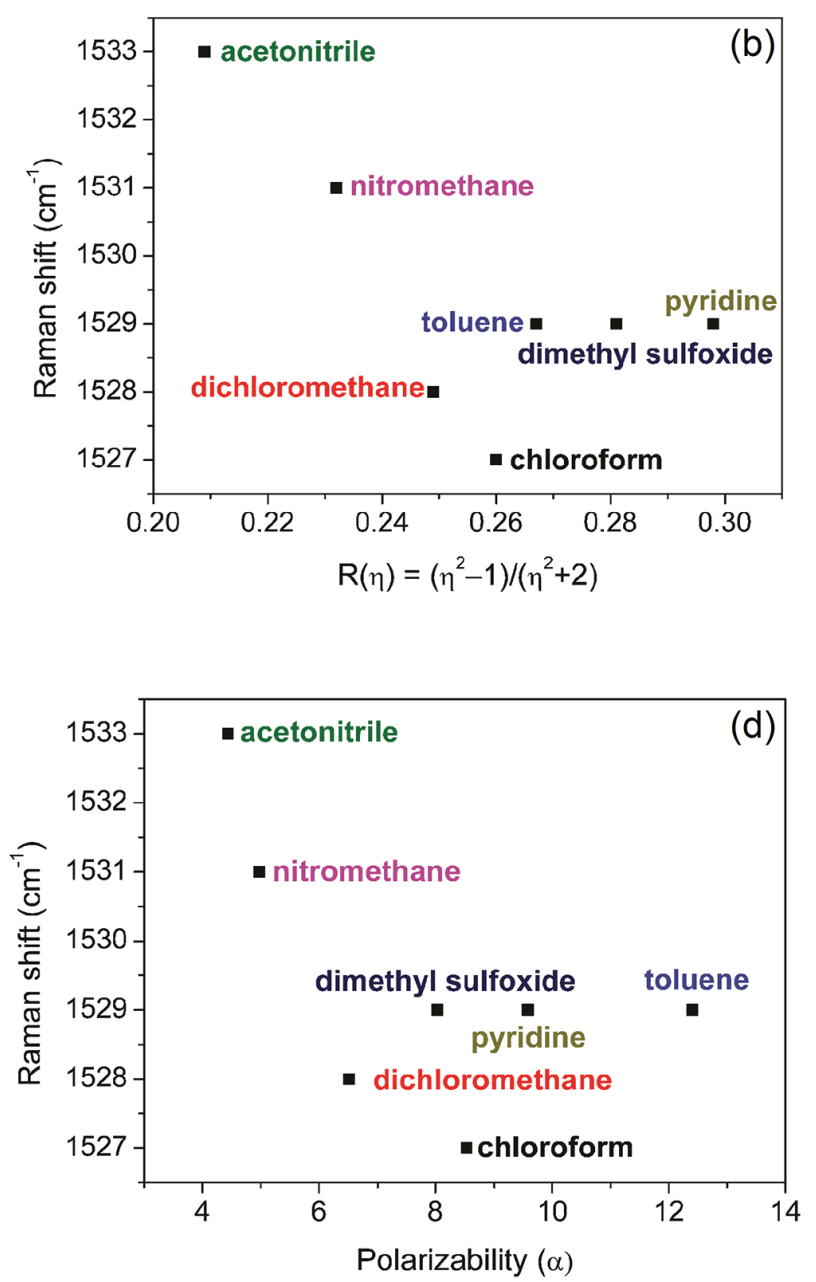

Figure 5. Correlation between solvent polarizability defined as R( $\eta$ ) with (a) the $S_{0} \rightarrow S_{2}$ electronic transition and (b) the Raman shifts, as well the correlation between solvent polarizability defined as $(\alpha)$ with (c) the $S_{0} \rightarrow S_{2}$ electronic transition and (d) the Raman shifts. 
observed in Raman shifts of bixin in different solvents could be correlated with the short extent of the conjugated double bonds and their effective delocalized pi-electrons (central portion only). The Raman spectrum obtained here for solid bixin, using infrared excitation, i.e., very far from any resonance with the excited electronic state, shows that only the $v_{1}(\mathrm{C}=\mathrm{C})$ mode is disturbed by the interaction with the solvent molecules (Figure 3, Figures S1-S7, SI section). For the other two vibrational bands $v_{2}(\mathrm{C}-\mathrm{C})$ at 1155 and $\rho\left(\mathrm{C}-\mathrm{CH}_{3}\right) 1012 \mathrm{~cm}^{-1}$, no significant disturbance has been observed due to the solvent interactions with the carotenoid, which implies that only the pi-electrons are directly involved in the solvent association.

Previous works ${ }^{13,24}$ from our group demonstrated that the chemical environment is responsible for some observable changes in the carotenoid Raman spectra, mainly based on the shift of the vibrational bands in the spectra. Here, the data that have been shown appear to show the same conclusion but based on different assumptions. Bixin in different solvents presented significant Raman shifts of approximately $9 \mathrm{~cm}^{-1}$ for the $v_{1}$ mode when compared to the solid sample. Nevertheless, subtle differences were observed between polar solvents such as acetonitrile, nitromethane and dimethyl sulfoxide (Figures S1, S2 and S4, SI section) and a non-polar solvent such as toluene (Figure S7, SI section). In dimethyl sulfoxide, which presents the highest dielectric constant value used in our studies (Table 1), the shift observed in the Raman spectrum is the same as that observed for the solvent with the lowest dielectric constant, namely toluene, as well the values of $S_{0} \rightarrow S_{2}$ transitions for bixin in each solvent. The other vibrational modes, as mentioned before, have not been disturbed by the interaction with any of the solvents investigated here. According to the polarizability of the solvents tested, ranging from $\alpha=4.44$ to 12.4 , it could be observed that solvents with the lowest values (acetonitrile: 4.44 and nitromethane: 4.97) showed the highest shifting of the Raman bands when compared to the solid bixin sample, i.e., from 1533 in solution to $1520 \mathrm{~cm}^{-1}$ in solid state (Table 1, Figures S1 and S2, SI section). Differences in the electronic transitions of the bixin in both solvents were also observed, showing a hypsochromic shifting in relation to the other solvents (Figure 2). As the solvents' polarizabilities increase, smaller differences in the Raman shifts are observed (Figures S3 to S7, SI section), indicating that the solvation of the solute molecules becomes more effective.

In order to better understand the influence of the solvent molecules on the dissolved cis-bixin, the Raman spectra were calculated considering two explicit solvent molecules in addition to the implicit model IEFPCM. For that DMSO and $\mathrm{CHCl}_{3}$ were used. Both are aprotic polar solvents which efficiently solubilize cis-bixin; ${ }^{30} \mathrm{CHCl}_{3}$ is the most efficient solvent for the quantitative extraction of bixin, ${ }^{29}$ whereas DMSO is commonly used to solubilize compounds in biological assays. ${ }^{58-60}$ The analyses were performed with an increasing number of solvent molecules in which each one has been inserted in different orientations as shown in Figures $6 \mathrm{~A}$ and $6 \mathrm{~B}$.

The presence of explicit molecules does not affect the overall profile of the cis-bixin spectra with $\mathrm{CHCl}_{3}$ molecules. The three bands at 1568,1525 and $1500 \mathrm{~cm}^{-1}$ have been assigned to the $\mathrm{C}=\mathrm{C}$ stretching mode, however, the most intense at $1525 \mathrm{~cm}^{-1}$ has a strong contribution of $\mathrm{C}-\mathrm{H}$ bending (rocking). Experimental data showed a strong band at $1527 \mathrm{~cm}^{-1}$ which matches with the calculated Raman spectrum; however, comparison of the results from both experiments (calculated and experimental) showed an upshifting of ca. $7 \mathrm{~cm}^{-1}$ in relation to the solid sample at $1520 \mathrm{~cm}^{-1}$ (Figure S5, SI section). cis-Bixin surrounded by four molecules of $\mathrm{CHCl}_{3}(\mathrm{Cl}-4 \mathrm{e})$ showed Raman bands shifting in the range of 4 to $2 \mathrm{~cm}^{-1}$ when compared with cis-bixin in the presence of one and two solvent molecules (Cl-1a, $1 \mathrm{~b}$ to $\mathrm{Cl}-2 \mathrm{c}, 2 \mathrm{~d}$, Figure 6). In contrast, the inclusion of DMSO molecules promoted a significant Raman band shift and also caused a band splitting at low wavenumbers, as can be seen in Figure 6B. The major differences were observed with Dm-1a and Dm-1b, containing one solvent molecule each. When the solvent is inserted close to the ester group at C-20 (Dm-1a), the intensity of the vibrational modes $\delta(\mathrm{C}-\mathrm{H})$ corresponding to $\mathrm{C}-14, \mathrm{C}-15$ and $\mathrm{C}-16$ at $1198 \mathrm{~cm}^{-1}$ is enhanced. However, in the Dm- $1 \mathrm{~b}$ case, where the solvent is close to the acid group, bands at 1198 and $1118 \mathrm{~cm}^{-1}$ are absent and bands at $1176,1165 \mathrm{~cm}^{-1} v(\mathrm{C}-\mathrm{C})$ from $\mathrm{C}-8$ to $\mathrm{C}-11$ are enhanced, all being assigned to the carbon-hydrogen rocking mode of vibration. Comparison of the one molecule solvent experiment (Dm-1a and Dm-1b) with the two and four solvent molecule analogues (Dm-2c, Dm-2d and Dm-4e) also showed differences in band position, mainly the $\delta\left(\mathrm{CH}_{3}\right)$ mode, which is shifted from $1446 \mathrm{~cm}^{-1}$ to higher wavenumbers at $1458 \mathrm{~cm}^{-1}$. Bands around 1565 and $1500 \mathrm{~cm}^{-1}$, which are attributed to $v(\mathrm{C}=\mathrm{C})$ stretching, were observed at similar wavenumbers in all five experiments (Figure 6B). These results clearly demonstrated that cis-bixin is soluble in both solvents by different mechanisms of solvation which does not seem to involve direct interactions between the selected solvents and the $\mathrm{C}=\mathrm{C}$ stretching modes. This also can be understood in relation to the pi electronic cloud of the backbone structure, since the pi electrons are not disturbed enough to be seen in the Raman spectrum as a shifted vibrational mode. Such a result can be corroborated by the electronic spectra shown in Figure 1, since the electronic transitions observed for cisbixin solutions in $\mathrm{CHCl}_{3}$ and DMSO are almost the same. 
(A)

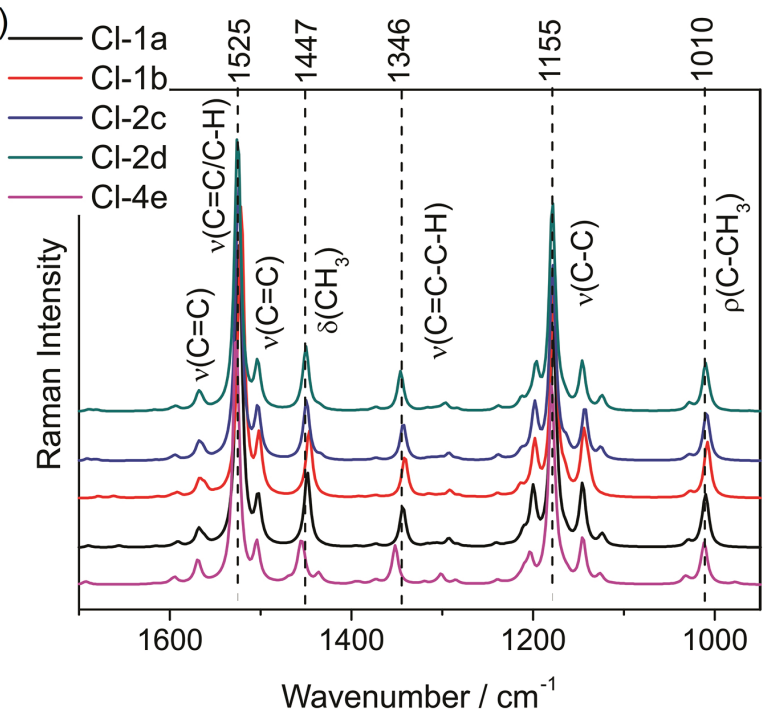

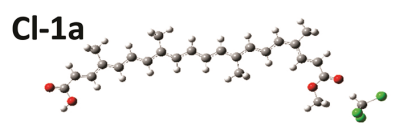

Cl-1b

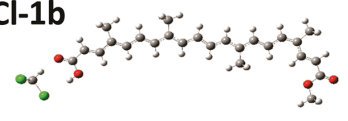
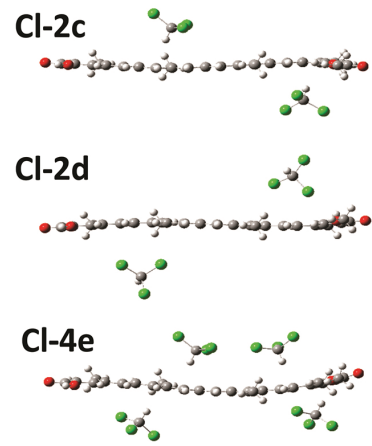

(B)
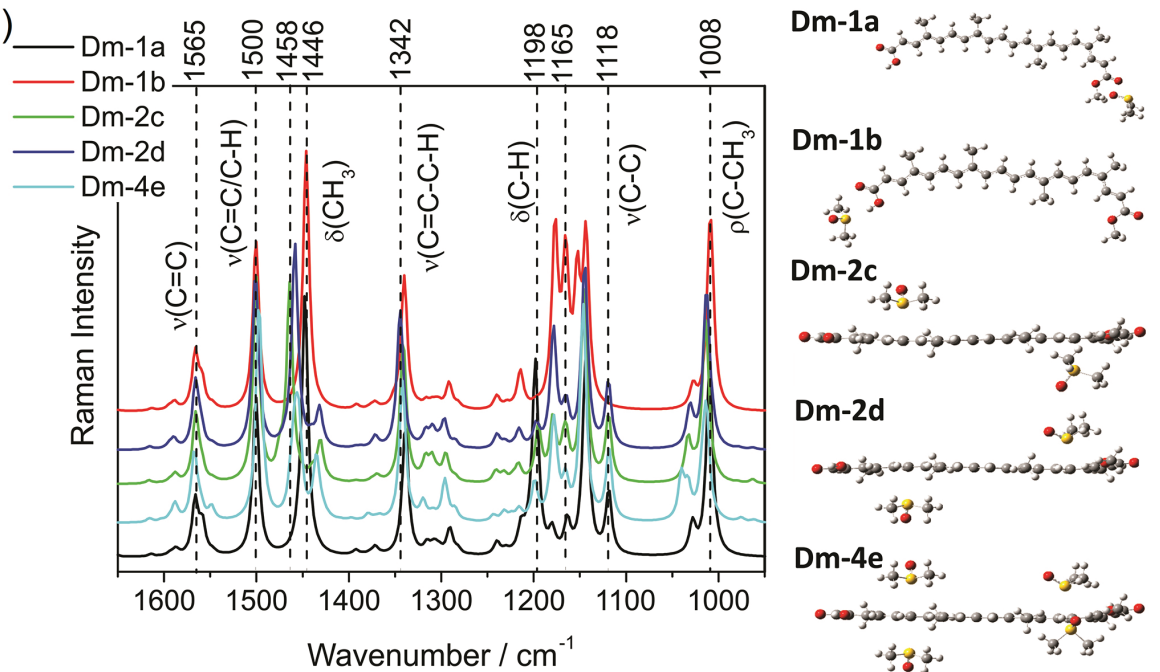

Dm-1b

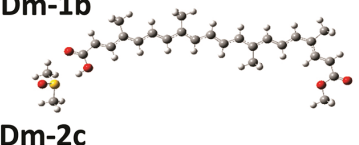

Dm-2c

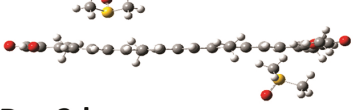

Dm-2d
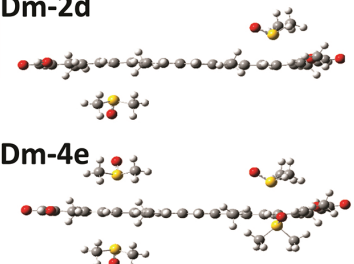

Figure 6. Calculated Raman spectrum of cis-bixin in $\mathrm{CHCl}_{3}$ and DMSO. The explicit solvent molecules (A) of $\mathrm{CHCl}_{3}$ positions (from $\mathrm{Cl}-1 \mathrm{a}$ to $\mathrm{Cl}-4 \mathrm{e}$ ) and (B) of DMSO (from Dm-1a to Dm-4e) are displayed. Both solvent explicit molecules were placed in different orientations (a-e): one (1) solvent molecule in the Cl-1a/Dm-1a and Cl-1b/Dm-1b figures; two (2) molecules in the Cl-2c/Dm-2c and Cl-2d/Dm-2d; and four (4) solvent molecules in the $\mathrm{Cl}-4 \mathrm{e} / \mathrm{Dm}-4 \mathrm{e}$ positions.

\section{Molecular dynamics}

Here, the results obtained from the molecular dynamics simulations (MD) undertaken using increased numbers of solvent molecules can now be reviewed. Due to the planar structure of cis-bixin, taking only one atom as a reference point for computing the radial distribution function (RDF) would provide a misleading result, since any selected atom of the molecule would also involve interaction with many solvent molecules with increasing distances. At this stage, therefore, one can consider five equally distinct points throughout the molecular structure and evaluate the RDF at each point. Then, from each one of these points, the distance between them and the solvation shell can be measured by extrapolating the approximation to only one distributed shell. Figure 7a shows the carbon atoms which were selected to perform this RDF analysis. The selected carbon atoms were: $\mathrm{C} 1, \mathrm{C} 5, \mathrm{C} 9, \mathrm{C} 13, \mathrm{C} 17$, and includes the carbon atom from the methyl ester group (Figure 7). The molecule has been numbered according to IUPAC nomenclature: (2E, $4 E, 6 E, 8 E, 10 E, 12 E, 14 E, 16 Z, 18 E)$-20-methoxy$4,8,13,17-\mathrm{te} t \mathrm{ra}$ m e th y $1-20-$ ox o i cos a $2,4,6,8,10,12,14,16,18$-nonaenoic acid, rather than using the common nomenclature established for carotenoids. The MD analysis has provided a good visualization of the solvation shells of the cis-bixin molecule surrounded by DMSO and $\mathrm{CHCl}_{3}$. Figures $7 \mathrm{~b}$ and $7 \mathrm{c}$ illustrate the first solvation shell obtained by RDF, where the cis-bixin can be seen surrounded by 70 DMSO molecules and 56 chloroform molecules, respectively.

Tables 2 and 3 provide the main details of the RDF results, including the number of molecules and radial 
(a)
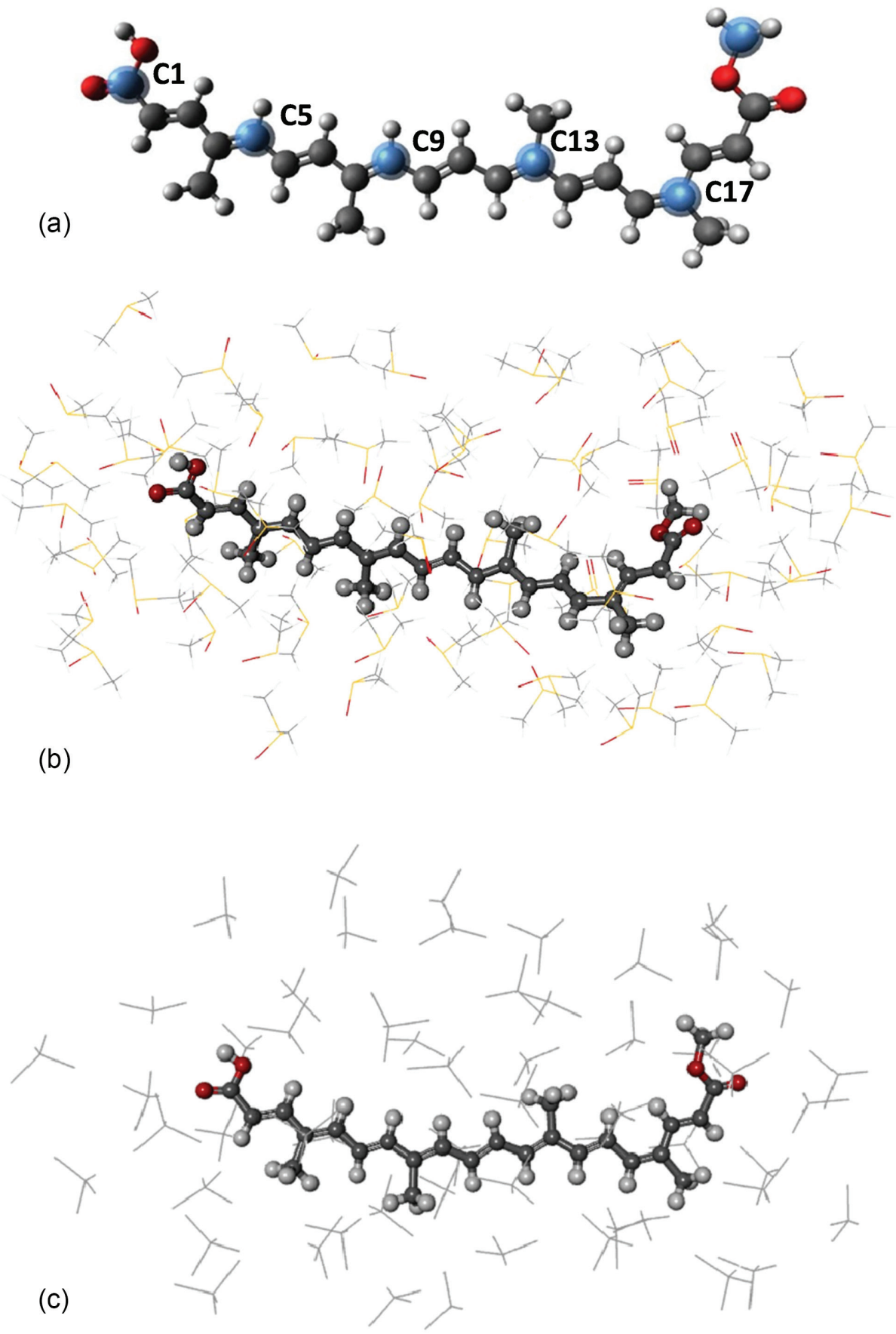

Figure 7. (a) cis-Bixin structure with highlighted carbon atoms to RDF analysis. From left to right: $\mathrm{C} 1$ (carboxyl group), C5, C9, C13, C17 and C20; (b) first solvation shell of $c$ is-bixin: 70 DMSO molecules are displayed as wires, while cis-bixin is shown as ball and stick model; (c) first solvation shell of cis-bixin: $56 \mathrm{CHCl}_{3}$ molecules are displayed as wires, while cis-bixin is shown as a ball and stick model.

distances around each carbon atom. It has been demonstrated that particular environments around each carbon may cause a difference in solvation. The data obtained from cis-bixin in DMSO have shown that the most exposed carbon atoms (methyl ester and C1) are less surrounded by solvent molecules with 8 and 11 molecules per carbon, respectively, since they are spatially available for the solvent to interact. On the other hand, the remaining carbon atoms have only two available sites for interaction, considering each face of the planar conformation of cis-bixin, so it is necessary for even more molecules to build the solvent shell (Table 2). This behavior is different for $c i s$-bixin in chloroform and the solvation number remains almost constant per carbon atom, with an average of solvent:cis-bixin molecules being 10:1. However, an anomalous behavior is seen for the number of chloroform molecules found to solvate 
Table 2. Main details about the methodology used to obtain the RDF results for cis-bixin in DMSO and solvent box information

\begin{tabular}{lcc}
\hline Information & Unit & $\rho^{*}\left(0.6022 \mathrm{M}^{-1}\right)$ \\
\hline Molecule $\left(\mathrm{M}^{1}\right)$ & 78.129 a.m.u & - \\
Density (calculated) & $1.1004 \mathrm{~g} \mathrm{~L}^{-1} / \mathrm{NTP}$ & 0.008481625 molecules $\AA^{-3}$ \\
\hline Selected atom & Radial distance / & Number of molecules \\
\hline C1 (carboxyl group) & 7.05 & 11 \\
C5 & 7.65 & 13 \\
C9 & 7.65 & 13 \\
C13 & 7.65 & 13 \\
C17 & 7.35 & 12 \\
C20 (methyl ester) & 6.15 & 8 \\
\hline
\end{tabular}

$\rho^{*}$ : factor for relative energy comparison; ${ }^{1} \mathrm{M}$ : molar mass; NTP: normal temperature and pressure.

Table 3. Main details about the methodology used to obtain the RDF results for $\mathrm{cis}$-bixin in chloroform and solvent box information

\begin{tabular}{lcc}
\hline Information & Unit & $\rho^{*}\left(0.6022 \mathrm{M}^{-1}\right)$ \\
\hline Molecule $\left(\mathrm{M}^{1}\right)$ & 119.369 a.m.u. & - \\
Density (calculated) & $1.49 \mathrm{~g} \mathrm{~cm}^{-3} / \mathrm{NTP}$ & 0.007516843 molecules $\AA^{-3}$ \\
\hline Selected atom & Radial distance / $\AA$ & Number of molecules \\
\hline C1 (carboxyl group) & 7.05 & 10 \\
C5 & 6.45 & 6 \\
C9 & 7.35 & 10 \\
C13 & 7.35 & 10 \\
C17 & 7.35 & 11 \\
C20 (methyl ester) & 6.75 & 9 \\
\hline
\end{tabular}

$\rho^{*}$ : factor for relative energy comparison; ${ }^{1} \mathrm{M}$ : molar mass; NTP: normal temperature and pressure.

C5 of cis-bixin. Methyl groups attached to carbons C4 and $\mathrm{C} 8$ may not cause a steric hindrance on the solvation shell of C5 surrounded by 6 solvent molecules, while the C9 experiences an environment where methyl group at $\mathrm{C} 8$ and $\mathrm{C} 13$ are on opposite sides, where the solvation shell shows the presence of 9 molecules (Table 3). These results demonstrate a different correlation between the geometry of part of the cis-bixin backbone with DMSO and $\mathrm{CHCl}_{3}$ solvent molecules. Despite both solvents have similar polarizability values they possess very different dielectric constants, which could explain the differences observed in solvation around the cis-bixin. According to Rahmalia et al., ${ }^{30}$ the extension of the bixin conjugated chain may be responsible for the preponderance of solute-solvent interactions which were more pronounced in DMSO and $\mathrm{CHCl}_{3}$. The absorbance at $\lambda_{\max }$ of bixin increased linearly with increasing concentration of aprotic polar solvents mainly in $\mathrm{CHCl}_{3}$. However, in our approach, cis-bixin seems to better interact with DMSO rather than
$\mathrm{CHCl}_{3}$ in terms of solvation. In other words, in the DMSO system more solvent molecules are required for the first solvent shell, which better solubilizes the solute.

These calculations have also shown that the cis-bixin molecules tend to adapt to the medium in order to minimize the chemical or physical interactions with the host solvent. From our preliminary energy calculations using the Poisson-Boltzmann method, implemented in Amber, this can also be observed, where it was found that an average solvation energy of $-20.5 \pm 0.6$ and $-13.4 \pm 0.5 \mathrm{kcal} \mathrm{mol}^{-1}$, for DMSO and $\mathrm{CHCl}_{3}$, respectively, were calculated.

It is important to point out that the change in wavenumber position of the $\mathrm{C}=\mathrm{C}$ bond vibration observed in the solid state compared with the sample in solution is not particular to bixin; a series of different polyenic systems, such as other carotenes ${ }^{26}$ as well conjugated polyenals have shown a similar behavior. ${ }^{61}$ In this work, it has been shown that experimental observations and computational results combined can give a better explanation of the solvent/ cis-bixin interaction in solution. Several different solvents, with a range of dielectric constants, varying from polar to non-polar, have been used to show that this interaction affects the solute molecule, which may confer upon each cis-bixin/solvent system a particular reactivity.

\section{Conclusions}

Raman spectroscopy is a very powerful tool to characterize carotenoids and conjugated polyenes since the vibrational bands, mainly the $\mathrm{C}=\mathrm{C}$ stretching mode at ca. $1500-1540 \mathrm{~cm}^{-1}$, are generally sensitive to molecular interactions. In this work, vibrational analyses of experimental and calculated Raman spectra were used to investigate how selected solvents act as different chemical environments which can be experienced by the nor-carotenoid bixin. The solute-solvent interaction was evaluated by the analysis of the Raman shifts of bixin in seven solvents with different dielectric constants and polarizability values. The Raman spectra of solvated bixin showed significant bathochromic shifts in the wavenumber position of $v(\mathrm{C}=\mathrm{C})$ when compared with the solid state. However, among the solutions subtle differences were observed, the most significant of these was the bixin/ acetonitrile system, which contains the solvent with the second highest dielectric constant ( $\varepsilon_{35.68)}$ and the lowest polarizability value ( $\alpha 4.44)$. As the polarizability of the solvents increased smaller differences in these Raman shifts were observed and no linear correlation was observed among solvents with different dielectric constant. Our results demonstrated that the polarizability of the solvent seems to be a more appropriate parameter to explain the 
differences in Raman shifts which could be correlated with the solvation degree of bixin. $\mathrm{CHCl}_{3}$ and DMSO are the solvents that best solubilize carotenoids and the theoretical Raman spectra of cis-bixin, which considered explicit solvent molecules in addition to the implicit model, showed that the number and the position of the $\mathrm{CHCl}_{3}$ molecules around the solute does not cause significant vibrational perturbation. However, in the DMSO experiment considerable changes in spectral profile could be observed when the solvent molecules are positioned close to the carbon-carbon backbone or close to the two end groups comprising the carboxylic acid (C-1) and ester (C-20) moieties. The solvent interaction with the polar portion of the solute is more efficient with DMSO than $\mathrm{CHCl}_{3}$. The radial distribution function (RDF) demonstrated that particular environments around each carbon of cisbixin may cause difference in solvation by DMSO and $\mathrm{CHCl}_{3}$. These particular differences observed do not seem to influence the Raman spectral features of cis-bixin in both solvents, since the solvation process involves large numbers of solvent molecules around the solute and the most intense vibrational modes overcome the minor ones. The Raman spectroscopic comparison between solid bixin with the solvated species showed that the perturbation of the $\pi$ electrons from the extended conjugation makes the $\mathrm{C}=\mathrm{C}$ stretching band a definitive marker for measuring the influence of chemical environment upon dissolved conjugated polyenes.

\section{Supplementary Information}

Supplementary data contain the experimental Raman spectra for cis-bixin, pure solvent and the solution of cis-bixin into the respective solvent (Figures S1 to S7), the heating step process from 9 to $309 \mathrm{~K}$ (not reached, Figure $\mathrm{S} 8$ ), and the potential energy $\left(\mathrm{E}_{\mathrm{pot}}\right)$, kinetic energy $\left(E_{t o t}\right)$ and total energy $\left(E_{t o t}\right)$ of the heating system (Figure S10). Also, Table S1 provides the $x y z$ spatial coordinates of the cis-bixin optimized structure (in DMSO-PCM). These data are available free of charge at http://jbcs.sbq.org.br as PDF file.

\section{Acknowledgments}

Authors are grateful to CNPq, CAPES and FAPEMIG (CEX-APQ-02068-14, CEX-APQ-00591-15) for financial support. This work is a collaboration research project of members of the Rede Mineira de Química (RQMG) supported by FAPEMIG. L. A. S. C. and L. F. C. O. thank $\mathrm{CNPq}$ for the award of research grants.

\section{Author Contributions}

Lenize F. Maia was responsible for investigation (Raman) and writing original draft; Isabela M. R. Rimulo was responsible for investigation (Raman and UV-Vis measurements); Vanessa E. de Oliveira was responsible for investigation (Raman and UV-Vis), visualization and writing original draft; Júlio A. F. Arvellos and Luiz Antônio S. Costa were responsible for investigation (electronic calculations) and software application; Howell G. M. Edwards and Luiz F. C. de Oliveira were responsible for writing original draft, review and editing the manuscript.

\section{References}

1. Yasushi, K.; J. Photochem. Photobiol., B 1991, 9, 265.

2. Frank, H. A.; Violette, C. A.; Trautman, J. K.; Shreve, A. P.; Owens, T. G.; Albrecht, A. C.; Pure Appl. Chem. 1991, 1, 109.

3. Jeevarajan, A. S.; Kispert, L. D.; Chumanov, G.; Zhou, C.; Cotton, T. M.; Chem. Phys. Lett. 1996, 259, 515.

4. Polívka, T.; Chábera, P.; Kerfeld, C. A.; Biochim. Biophys. Acta 2013, 1827, 248.

5. Kish, E.; Pinto, M. M. M.; Kirilovsky, D.; Spezia, R.; Robert, B.; Biochim. Biophys. Acta 2015, 1847, 1044.

6. Krinsky, N. I.; Free Radical Biol. Med. 1989, 7, 617.

7. Noguchi, T.; Hayashi, H.; Tasumi, M.; Atkinson, G. H.; J. Phys. Chem. 1991, 95, 3167.

8. Rimai, L.; Kilponen, R. G.; Gill, D.; J. Am. Chem. Soc. 1970, 92, 3824.

9. Gill, D.; Kilponen, R. G.; Rimai, L.; Nature 1970, 227, 743.

10. Rimai, L.; Heyde, M. E.; Gill, D.; J. Am. Chem. Soc. 1973, 95 , 4493.

11. Hashimoto, H.; Koyama, Y.; Hirata, Y.; Mataga, N.; J. Phys. Chem. 1991, 95, 3072.

12. Thrash, R. J.; Fang, H. L. B.; Leroi, G. E.; J. Chem. Phys. 1977, 67, 5930.

13. de Oliveira, V. E.; Castro, H. V.; Edwards, H. G. M.; de Oliveira, L. F. C.; J. Raman Spectrosc. 2010, 41, 642.

14. Schaffer, H. E.; Chance, R. R.; Silbey, R. J.; Knoll, K.; Schrock, R. R.; J. Chem. Phys. 1991, 94, 4161.

15. Brambilla, L.; Tommasini, M.; Zerbi, G.; Stradi, R.; J. Raman Spectrosc. 2012, 43, 1449.

16. Ferreira, B. S.; de Almeida, C. G.; Le Hyaric, M.; de Oliveira, V. E.; Edwards, H. G. M.; de Oliveira, L. F. C.; Spectrosc. Lett. 2013, 46, 122.

17. de Oliveira, V. E.; Miranda, M.; Soares, M. C. S.; Edwards, H. G. M.; de Oliveira, L. F. C.; Spectrochim. Acta, Part A 2015, 150,373 .

18. de Oliveira, L. N.; de Oliveira, V. E.; D’Avila, S.; Edwards, H. G. M.; de Oliveira, L. F. C.; Spectrochim. Acta, Part A 2013, 114,541 . 
19. Karampelas, S.; Fritsch, E.; Mevellec, J. Y.; Sklavounos, S.; Soldatos, T.; Eur. J. Mineral. 2009, 21, 85.

20. Maia, L. F.; Fleury, B. G.; Lages, B. G.; Barbosa, J. P.; Pinto, A. C.; Castro, H. V.; de Oliveira, V. E.; Edwards, H. G. M.; de Oliveira, L. F. C.; J. Raman Spectrosc. 2011, 42, 653.

21. Maia, L. F.; de Oliveira, V. E.; de Oliveira, M. E. R.; Fleury, B. G.; de Oliveira, L. F. C.; J. Raman Spectrosc. 2012, 43, 161.

22. Maia, L. F.; de Oliveira, V. E.; de Oliveira, M. E. R.; Reis, F. D.; Fleury, B. G.; Edwards, H. G. M.; de Oliveira, L. F. C.; J. Raman Spectrosc. 2013, 44, 560.

23. Maia, L. F.; Ferreira, G. F.; Costa, R. C. C.; Lucas, N. C.; Teixeira, R. I.; Fleury, B. G.; Edwards, H. G. M.; de Oliveira, L. F. C.; J. Phys. Chem. A 2014, 118, 3429.

24. de Oliveira, V. E.; Almeida, E. W. C.; Castro, H. V.; Edwards, H. G. M.; dos Santos, H. F.; de Oliveira, L. F. C.; J. Phys. Chem. A 2011, 115, 8511.

25. Mendes-Pinto, M. M.; Sansiaume, E.; Hashimoto, H.; Pascal, A. A.; Gall, A.; Robert, B.; J. Phys. Chem. B 2013, 117, 11015.

26. Macernis, M.; Sulskus, J.; Malickaja, S.; Robert, B.; Valkunas, L.; J. Phys. Chem. A 2014, 118, 1817.

27. Vilar, D. A.; Vilar, M. S. A.; Moura, T. F. A. L.; Raffin, F. N.; Oliveira, M. R.; Franco, C. F. O.; Athayde-Filho, P. F.; Diniz, M. F. F. M.; Barbosa-Filho, J. M.; Sci. World J. 2014, article ID 857292.

28. Scotter, M.; Food Addit. Contam., Part A 2009, 26, 1123.

29. Giridhar, P.; Venugopalan, A.; Parimalan, R.; J. Sci. Res. Rep. 2014, 3, 327.

30. Rahmalia, W.; Fabre, J. F.; Usman, T.; Mouloungui, Z.; Spectrochim. Acta, Part A 2014, 131, 455.

31. Islam, S.; Rather, L. J.; Mohammad, F.; J. Adv. Res. 2016, 7, 499.

32. Rivera-Madrid, R.; Aguilar-Espinosa, M.; Cárdenas-Conejo, Y.; Garza-Caligaris, L. E.; Front. Plant Sci. 2016, 7, 1406.

33. Gomez-Ortíz, N. M.; Vazquez-Maldonado, I. A.; Perez-Espadas, A. R.; Mena-Rejon, G. J.; Azamar-Barrios, J. A.; Oskam, G.; Sol. Energy Mater. Sol. Cells 2010, 94, 40.

34. Haryanto, D. A.; Landuma, S.; Purwanto, A.; AIP Conf. Proc. 2014, 1586, 104.

35. Llansola-Portoles, M. J.; Sobotka, R.; Kish, E.; Shukla, M. K.; Pascal,A.A.; Polívka,T.; Robert, B.; J. Biol. Chem. 2017,292, 1396.

36. Dobrowolski, J. In Carotenoids: Nutrition, Analysis and Technology, $1^{\text {st }}$ ed.; Kaczor, A.; Baranska, M., eds.; John Wiley and Sons: Chichester, 2016, ch. 6.

37. Reszczynska, E.; Welc, R.; Grudzinski, W.; Trebacz, K.; Gruszecki, W. I.; Arch. Biochem. Biophys. 2015, 584, 125.

38. de Oliveira, L. F. C.; Dantas, S. O.; Velozo, E. S.; Santos, P. S.; Ribeiro, M. C. C.; J. Mol. Struct. 1997, 435, 101.

39. Grimme, S.; J. Comput. Chem. 2004, 25, 1463.

40. Grimme, S.; J. Comput. Chem. 2006, 27, 1787.

41. Krishnan, R.; Binkley, J. S.; Seeger, R.; Pople, J. A.; J. Chem. Phys. 1980, 72, 650.
42. Cossi, M.; Barone, V.; Cammi, R.; Tomasi, J.; Chem. Phys. Lett. 1996, 255, 327.

43. Barone, V.; Cossi, M.; Tomasi, J.; J. Chem. Phys. 1997, 107, 3210.

44. Frisch, M. J.; Trucks, G. W.; Schlegel, H. B.; Scuseria, G. E.; Robb, M. A.; Cheeseman, J. R.; Scalmani, G.; Barone, V.; Petersson, G. A.; Nakatsuji, H.; Li, X.; Caricato, M.; Marenich, A.; Bloino, J.; Janesko, B. G.; Gomperts, R.; Mennucci, B.; Hratchian, H. P.; Ortiz, J. V.; Izmaylov, A. F.; Sonnenberg, J. L.; Williams-Young, D.; Ding, F.; Lipparini, F.; Egidi, F.; Goings, J.; Peng, B.; Petrone, A.; Henderson, T.; Ranasinghe, D.; Zakrzewski, V. G.; Gao, J.; Rega, N.; Zheng, G.; Liang, W.; Hada, M.; Ehara, M.; Toyota, K.; Fukuda, R.; Hasegawa, J.; Ishida, M.; Nakajima, T.; Honda, Y.; Kitao, O.; Nakai, H.; Vreven, T.; Throssell, K.; Montgomery Jr., J. A.; Peralta, J. E.; Ogliaro, F.; Bearpark, M.; Heyd, J. J.; Brothers, E.; Kudin, K. N.; Staroverov, V. N.; Keith, T.; Kobayashi, R.; Normand, J.; Raghavachari, K.; Rendell, A.; Burant, J. C.; Iyengar, S. S.; Tomasi, J.; Cossi, M.; Millam, J. M.; Klene, M.; Adamo, C.; Cammi, R.; Ochterski, J. W.; Martin, R. L.; Morokuma, K.; Farkas, O.; Foresman, J. B.; Fox, D. J.; Gaussian 09, Revision A.02; Gaussian, Inc., Wallingford, CT, 2016.

45. Wang, J.; Wolf, R. M.; Caldwell, J. W.; Kollman, P. A.; Case, D. A.; J. Comput. Chem. 2004, 25, 1157.

46. Sprenger, K. G.; Jaeger, V. W.; Pfaendtner, J.; J. Phys. Chem. B 2015, 119, 5882 .

47. Case, D. A.; Darden, T. A.; Cheatham III, T. E.; Simmerling, C. L.; Wang, J.; Duke, R. E.; Luo, R.; Walker, R. C.; Zhang, W.; Merz, K. M.; Roberts, B.; Hayik, S.; Roitberg, A.; Seabra, G.; Swails, J.; Götz, A. W.; Kolossváry, I.; Wong, K. F.; Paesani, F.; Vanicek, J.; Wolf, R. M.; Liu, J.; Wu, X.; Brozell, S. R.; Steinbrecher, T.; Gohlke, H.; Cai, Q.; Ye, X.; Wang, J.; Hsieh, M.-J.; Cui, G.; Roe, D. R.; Mathews, D. H.; Seetin, M. G.; Salomon-Ferrer, R.; Sagui, C.; Babin, V.; Luchko, T.; Gusarov, S.; Kovalenko, A.; Kollman, P. A.; AMBER 12; University of California, San Francisco, USA, 2012.

48. Fox, T.; Collman, P.; J. Phys. Chem. B 1998, 102, 8070.

49. Britton, G.; Liaaen-Jensen, S.; Pfander, H. In Carotenoids; Britton, G.; Liaaen-Jensen, S.; Pfander, H., eds.; Birkhauser Verlag: Basel, Switzerland, 1995, p. 329.

50. Schulz, H.; Baranska, M.; Baranski, R.; Biopolymers 2005, 77, 212.

51. Tay-Agbozo, S.; Street, S.; Kispert, L. D.; J. Photochem. Photobiol., A 2018, 362, 31.

52. Ishigaki, M.; Meksiarun, P.; Kitahama, Y.; Zhang, L.; Hashimoto, H.; Genkawa, T.; Ozaki, Y.; J. Phys. Chem. B 2017, $121,8046$.

53. Subramanian, B.; Tchoukanova, N.; Djaoued, Y.; Pelletierb, C.; Ferron, M.; J. Raman Spectrosc. 2013, 44, 219.

54. Wang, C.; Berg, C. J.; Hsu, C.; Merrill, B. A.; Tauber, M. J.; J. Phys. Chem. B 2012, 116, 10617. 
55. Bosque, R.; Sales, J.; J. Chem. Inf. Comput. Sci. 2002, 42, 1154.

56. Llansola-Portoles, M. J.; Pascal, A. A.; Robert, B.; J. R. Soc., Interface 2017, 14, 20170504.

57. Andersson, P. O.; Gillbro, M.; Ferguson, L.; Gogdell, R. J.; Photochem. Photobiol. 1991, 9, 353.

58. Antunes, L. M. G.; Pascoal, L. M.; Bianchi, M. L. P.; Dias, F. L.; Mutat. Res., Genet. Toxicol. Environ. Mutagen. 2005, 585, 113.
59. Santos, G. C.; Mendonça, L. M.; Antonucci, G. A.; Santos, A. C.; Antunes, L. M. G.; Bianchi, M. L. P.; Food Chem. Toxicol. 2012, 50, 335.

60. Lin, H. W.; Chang, T. J.; Yang, D. J.; Chen, Y. C.; Wang, M.; Chang, Y. Y.; Food Chem. 2012, 134, 2169.

61. Milani, A.; Zoppo, M.; Tommasini, M.; Zerbi, G.; J. Phys. Chem. B 2008, 112, 1619.

Submitted: September 20, 2019

Published online: February 6, 2020 